\title{
Spectrum of neurodevelopmental disabilities in children with cerebellar malformations
}

\author{
MARIE-EVE BOLDUC ${ }^{1}$ | ADRÉ J DU PLESSIS ${ }^{2,3}$ | NANCY SULLIVAN ${ }^{4}$ | OMAR S KHWAJA ${ }^{2}$ | XUN ZHANG ${ }^{5}$ | \\ KATHERINE BARNES ${ }^{2}$ | RICHARD L ROBERTSON ${ }^{6}$ | CATHERINE LIMPEROPOULOS ${ }^{1,2,7,8,9}$
}

1 School of Physical and Occupational Therapy, McGill University, Montreal, Quebec, Canada. 2 Fetal-Neonatal Neurology Research Program, Department of Neurology, Children's Hospital Boston and Harvard Medical School, Boston, MA, USA. 3 Division of Fetal and Transitional Medicine, Children's National Medical Center, George Washington University, Washington, DC, USA. 4 Developmental Medicine Center, Children's Hospital Boston and Harvard Medical School, Boston, MA, USA. 5 Research Institute, McGill University Health Centre, Montreal, Quebec, Canada. 6 Department of Radiology, Children's Hospital Boston and Harvard Medical School, Boston, MA, USA. 7 Department of Neurology and Neurosurgery, Montreal Children's Hospital, McGill University, Montreal, Quebec, Canada. 8 Department of Pediatrics, Montreal Children's Hospital, McGill University, Montreal, Quebec, Canada. 9 Division of Diagnostic Imaging and Radiology, Children's National MedicalCenter, George Washington University, Washington, DC, USA.

Correspondence to Dr Catherine Limperopoulos at Division of Diagnostic Imaging and Radiology, Children's National Medical Center, 111 Michigan Ave NW, Washington, DC 20010, USA. E-mail: climpero@cnmc.org

This article is commented on by Poretti on page 389 of this issue.

\section{PUBLICATION DATA}

Accepted for publication 22nd December 2010.

Published online 18th March 2011.

\begin{tabular}{ll}
\multicolumn{2}{l}{ ABBREVIATIONS } \\
CBCL & Child Behavior Checklist \\
CBM & Cerebellar malformation \\
DWM & Dandy-Walker malformation \\
ICVH & Inferior cerebellar vermis hypoplasia \\
M-CHAT & Modified Checklist for Autism \\
& Toddlers \\
PDMS & $\begin{array}{l}\text { Peabody Developmental Motor } \\
\end{array}$ \\
Seales \\
PedsOL & Pediatric Quality of Life Inventory \\
QOL & Quality of life \\
VABS & Vineland Adapted Behavior Scale \\
& \\
\hline
\end{tabular}

AlM Advances in perinatal care and neuroimaging techniques have increased the detection of cerebellar malformations (CBMs) in the fetus and young infant. As a result, this has necessitated a greater understanding of the neurodevelopmental consequences of CBMs on child development. The aim of this study was to delineate the impact of CBMs on long-term neurodevelopmental outcomes.

METHOD We conducted a cross-sectional study and systematically identified children with CBMs born between December 2000 and December 2006. We then performed follow-up magnetic resonance imaging studies, neurologic examination, and standardized neurodevelopmental outcome testing (Mullen Scales of Early Learning, Vineland Adaptive Behavior Scale, Child Behavior Checklist, Modified Checklist for Autism in Toddlers, and the Pediatric Quality of Life Inventory). RESULTS Our sample comprised 49 children ( 29 males, 20 females; mean age, $28.4 \mathrm{mo}$, SD 16.4) with a CBM. Infants with evidence of acquired fetal or neonatal brain injury, intracranial birth trauma, inherited metabolic disease, or major pre- or postnatal cerebral ischemia were excluded. Our findings highlight that children with CBMs experience a high prevalence of neurologic, developmental, and functional disabilities including motor, cognitive, language, and social-behavioral deficits, as well as poor quality of life. The associated supratentorial anomalies, chromosomal findings, and malformations affecting the cerebellar vermis were significant independent predictors of neurodevelopmental disabilities in young children with CBMs. The associated supratentorial anomalies and chromosomal findings were also predictive of global developmental delay $(p=0.01)$, cognitive impairment $(p=0.03)$, gross and fine motor delay $(p=0.02$ and $p=0.01$ respectively), and positive screening for autism spectrum disorder $(p=0.01)$. Additionally, malformations affecting the cerebellar vermis were significant independent predictors of expressive language ( $p=0.04$ ) and gross motor delays $(p=0.02$ ).

INTERPRETATION Developmental surveillance and early intervention programs should be an integral part of the long-term follow-up of survivors of CBM.
Advances in perinatal care and brain imaging techniques have increased the detection and diagnostic accuracy of cerebellar malformations (CBMs) in infants and more recently in the fetus. These advances have necessitated a greater understanding of the impact of these early life lesions on brain and child development. However, the developmental and functional correlates of CBMs in young children remain poorly defined.

The role of the cerebellum as a center for motor coordination and execution was first described in the early 1800 s. ${ }^{1}$ For many years, clinical and research interest focused on the motor consequences of cerebellar injury. Although anecdotal reports of cognitive impairments in the setting of cerebellar lesions appeared in earlier years, more recent data in adults and older children have supported an important role for the cerebellum in the development of cognitive and social functions, thereby calling for a broader investigation of the functional consequences of cerebellar pathology. ${ }^{2-4}$

Available evidence of the outcome of young children with CBMs is controversial. Some studies suggest that CBMs are associated with significant neurodevelopmental morbidities, 
whereas others suggest a more favorable outcome. ${ }^{5}$ A recent systematic review concluded that studies ascertaining the outcome of children with CBMs have so far been methodologically flawed, because of a lack of rigorous study design, and largely limited to informal documentation of neurodevelopmental progress, resulting in poor outcome determination. ${ }^{5}$ Consequently, the spectrum of neurodevelopmental outcomes of children with CBMs remains poorly defined. The aim of this study was to examine the impact of CBMs on developmental and functional outcomes, as well as quality of life (QOL), in young children.

\section{METHOD}

\section{Procedures}

As part of a cross-sectional study, we identified children with a diagnosis of CBM born between December 2000 and December 2006 through a systematic electronic search of the magnetic resonance imaging (MRI) database of the Children's Hospital Boston, MA, USA. Infants born at term with an antenatal or neonatal diagnosis of Dandy-Walker malformation (DWM), cerebellar hypoplasia and/or vermis hypoplasia, rhombencephalosynapsis, and Joubert syndrome were selected. We also included children with mega cisterna magna or posterior fossa retrocerebellar cyst because these lesions may be difficult to distinguish from primary cerebellar anomalies. Infants with evidence of acquired fetal or neonatal brain injury, intracranial birth trauma, inherited metabolic disease, or major pre- or postnatal cerebral ischemia were excluded. Medical records were reviewed for pertinent demographic and clinical information including neonatal (e.g. gestational age, birthweight) and postnatal (e.g. seizures, genetic findings) factors. The study was approved by the Committee on Clinical Investigation at the Children's Hospital Boston. The child's parent or legal guardian provided written informed consent in all cases.

\section{MRI studies}

All children underwent MRI studies on a 1.5T General Electric System (GE-Medical Systems, Milwaukee, WI, USA). We acquired sagittal and axial spin-echo T1-weighted sequences, axial fast spin-echo $\mathrm{T} 2$-weighted sequences, and susceptibility sequences using a multiplanar gradient recall gradient-echo technique.

\section{Neuroimaging diagnostic criteria}

A pediatric neuroradiologist (RLR) who was blind to the clinical diagnosis reviewed all MRI studies to confirm the diagnosis. DWM was diagnosed when the following three criteria were met: (1) vermis agenesis or hypogenesis; (2) cystic dilatation of the fourth ventricle; and (3), an abnormally high tentorium with enlargement of the posterior fossa. We did not use the term 'Dandy-Walker variant' because the inconsistent application of this term has severely limited its utility. ${ }^{6}$ Inferior cerebellar vermis hypoplasia (ICVH) was diagnosed when caudal growth of the inferior vermis over the fourth ventricle was incomplete, as assessed on MRI using the midline sagittal plane. The term 'isolated ICVH' was used to indicate that the

\section{What this paper adds}

- A better understanding and definition of the prevalence of developmental disabilities in children with CBMs.

- It is the first of its kind to evaluate $\mathrm{OOL}$ in young children with CBMs.

- It has established an urgent need for better outcome definition in young survivors with CBMs.

- It identifies the need for early intervention services.

lower third of the cerebellar vermis was incomplete with normal-shaped or near-normal-shaped cerebellar hemispheres, a normal-sized posterior fossa, and normal supratentorial structures. Conversely, we diagnosed vermis hypoplasia when less than two-thirds of the cerebellar vermis had formed. Cerebellar hypoplasia was diagnosed as unilateral when one cerebellar hemisphere was underdeveloped (but with a normal vermis) or bilateral when both cerebellar hemispheres were small; the latter diagnosis invariably included vermis hypoplasia. A diagnosis of rhombencephalosynapsis was made when the vermis was absent and the cerebellar hemispheres were fused in the midline. Mega cisterna magna was diagnosed in patients with enlarged retrocerebellar space $(>10 \mathrm{~mm})$, presumably owing to a variance in skull growth, with an otherwise normal cerebellum. A posterior fossa retrocerebellar cyst was diagnosed when a cystic pouch behind the cerebellum was not in communication with the fourth ventricle. Finally, Joubert syndrome was diagnosed in the presence of the molar tooth sign on axial MRIs, which is characterized by an abnormally deep interpeduncular fossa, enlarged superior cerebellar peduncles that are more horizontally oriented, and a hypoplastic cerebellar vermis.

\section{Neurologic examination}

Two pediatric neurologists (AJDP and OSK) performed a formal neurologic examination. This included measurement of head circumference, and assessment of oculomotor, sensory, and motor functions (e.g. muscle tone, deep tendon reflexes, and gait). Microcephaly was defined as a head circumference $2 \mathrm{SDs}$ below the normative mean, corresponding to the third centile.

\section{Standardized outcome measures}

The following standardized assessments were used to characterize the spectrum of developmental disabilities in our cohort. All evaluators were blind to MRI findings, perinatal/neonatal complications, and each other's clinical findings.

\section{Developmental and functional measures}

The Mullen Scales of Early Learning (Mullen) ${ }^{7}$ was administered by a licensed child psychologist (NS). The Mullen Scales of Early Learning is a standardized, norm-referenced, developmental evaluation that includes five subscales: receptive and expressive language, visual reception, gross and fine motor skills, and an early learning composite quotient. The Peabody Developmental Motor Scales (PDMS) ${ }^{8}$ were administered by an occupational therapist (CL) to assess gross and fine motor function. The Vineland Adaptive Behavior Scale (VABS) ${ }^{9}$ was administered by a licensed child psychologist (NS). It is a discriminative, norm-referenced measure of functional status 
that assesses communication, daily living skills, socialization, and motor skills. A score below 2SDs of the normative mean was defined as a significant delay for the Mullen, PDMS, and VABS.

\section{Social-behavioral and Q0L measures}

The Child Behavior Checklist (CBCL) ${ }^{10}$ is a caregiver report that assesses behavioral and social difficulties. Scores of internalizing and externalizing behaviors were derived. The scores are expressed as $\mathrm{T}$ scores, and a score equal to or above 60 was defined as impaired. The Modified Checklist for Autism in Toddlers (M-CHAT) ${ }^{11}$ is a parental report that is used to evaluate the risk of autism spectrum disorders (ASDs). It is composed of 23 binary (yes/no) questions, among which six are critical items. Failing of three items in total or two critical items was used as a cut-off. Health-related QOL was assessed using the Pediatric Quality of Life Inventory 4.0 (PedsQL), ${ }^{12}$ that encompasses four domains: physical, emotional, social, and school/daycare. It uses parent proxy-report for children under 5 years of age. A cut-off of 65.4 was used to identify children with impaired QOL.

\section{Socio-economic and medical history data}

The Hollingshead Two Factor Index of Social Status ${ }^{13}$ was used to assess socio-economic status. A medial history questionnaire was also administered to characterize our sample (e.g. use of medications, presence of significant health problems).

\section{Statistical analysis}

Continuous clinical and developmental data were summarized using means and SDs, whereas categorical data were summarized using proportions. Continuous scores of primary outcomes were compared between diagnostic groups using the independent $t$-test (Mullen and PDMS), and a $\chi^{2}$ test was used for dichotomous data (M-CHAT). Our primary outcome measures included the Mullen (receptive and expressive language, visual reception, and early learning composite quotient), the PDMS (gross and fine motor scores), and the M-CHAT. Results of secondary outcome measures (VABS, CBCL, and PedsQL) were reported using descriptive statistics. Pearson's correlations were used to examine the bivariate relation between the various outcome measures.

Independent predictors of the seven primary outcomes were identified using multivariate analyses. The multivariate regression models included the presence of associated central nervous system (CNS) and/or chromosomal anomalies and the presence of malformations that involved the cerebellar vermis as independent predictors of outcome. Additionally, head circumference and age at testing were added to the model to control for their confounding effect on outcome. Socioeconomic status was not used as a confounder in our multivariate analyses because it was not significantly correlated with outcome. Linear regressions were used for the subscale scores of the Mullen and PDMS, and logistic regression was used for the M-CHAT. Residual plots were examined for fits of linear regressions, and the Hosmer-Lemshow test for logistic regressions.

\section{RESULTS}

We identified 59 children with a diagnosis of CBM who met our inclusion criteria. Of these, four died postnatally and three were lost to follow-up. We approached the remaining 52 families, of whom 49 (94\% enrolment rate) agreed to participate. Children (29 males, 20 females; age range 1-6y) were evaluated at a mean (SD) age of 28.4 months (16.4). All children were born at term (gestational age 39.0 [1.4] wks; birthweight $3290 \mathrm{~g}$ [250]). However, one child died before all outcome measures could be completed and some children did not complete all items of the neurologic examination owing to their young age at testing.

\section{Clinical diagnoses}

Nine different diagnostic groups of CBMs were identified in our study. Thirty-six children had isolated CBMs and 13 had associated CNS anomalies. Additionally, 39 children underwent chromosomal testing, of whom eight were found to have concomitant chromosome abnormalities or syndromes (Table I).

\section{Neurologic outcomes}

Thirty-nine of the 47 children who underwent neurologic examination showed neurologic abnormalities including impaired gait $(n=23)$, axial hypotonia $(n=22)$, appendicular hypotonia $(n=18)$, hypertonia $(n=6)$, and hyperactive tendon reflexes $(n=12)$. Furthermore, eye position or eye movement abnormalities were present in 17 out of 46 children, including nystagmus $(n=10)$, strabismus $(n=6)$, and oculomotor apraxia $(n=1)$. In addition, extraocular muscle palsy (4/44) was also documented. Upper motor neuron lesions were identified in 16 out of 47, and microcephaly was documented in 10 out of 47 children. Among the children who were old enough to be tested, impairments in finger-to-nose and rapid alternating movements were present in six out of 23 and five out of 11 respectively. Head titubation was present in 11 out of 47 of the children. Abnormal movement patterns included ataxia (8/46), upper extremity dyskinesias (5/46), and dystonia $(2 / 46)$.

\section{Associated medical conditions}

Vision and hearing problems were present in 21 out of 47 and three out of 47 children respectively. Eight children had shunts, of whom four had undergone shunt revisions. The children who required shunting procedures had the following cerebellar diagnoses: DWM $(n=5)$, posterior fossa retrocerebellar cyst $(n=2)$, and rhombencephalosynapsis $(n=1)$. Additionally, four children had seizures. Other significant health problems included gastro-esophageal reflux/vomiting $(n=4)$ and feeding problems $(n=5)$.

\section{Developmental and functional outcomes}

Fourteen children with CBMs had normal developmental scores. The diagnostic categories of the subgroup of children with normal developmental outcomes included the following: ICVH $(n=8)$, unilateral cerebellar hypoplasia $(n=2)$, posterior fossa retrocerebellar cyst $(n=2)$, DWM $(n=1)$, and mega 
Table I: Diagnostic categories for cerebellar malformation, associated CNS, and chromosomal findings

\begin{tabular}{|c|c|c|c|c|}
\hline Cerebellar malformation & $\begin{array}{l}\text { Frequency, } \\
\%(n=49)\end{array}$ & Associated CNS anomalies & Syndromes & $\begin{array}{l}\text { Genetic testing } \\
\text { (abnormal/tested) }\end{array}$ \\
\hline Inferior vermis hypoplasia & 35 & Dysgenesis of corpus callosum (3) & & $0 / 9$ \\
\hline \multirow{3}{*}{$\begin{array}{l}\text { A total of } 13 \text { children had supratentoral } \\
\text { anomalies however several children had } \\
\text { a combination of them (i.e, more than one) }\end{array}$} & & Abnormal cerebral gyral pattern (2) & & \\
\hline & & Periventricular white matter abnormalities (1) & & \\
\hline & & Ventriculomegaly (1) & & \\
\hline \multirow[t]{4}{*}{ Bilateral cerebellar hypoplasia } & 16 & Dysgenesis of corpus callosum (3) & Cri du chat (1) & $1 / 8(5 p 15.2)$ \\
\hline & & Cerebellar heterotopias (3) & CHARGE syndrome (1) & \\
\hline & & Abnormal cerebral gyral pattern (1) & & \\
\hline & & Decreased white matter ( 1 ) & & \\
\hline \multirow[t]{2}{*}{ Vermis hypoplasia (more extensive) } & 2 & Dysgenesis of corpus callosum (2) & CHARGE syndrome (1) & 1/6 (chromosome \\
\hline & & Periventricular white matter abnormalities (1) & & 10q deletion) \\
\hline Dandy-Walker malformation & 10 & Dysgenesis of corpus callosum (1) & & $0 / 4$ \\
\hline Unilateral cerebellar hypoplasia & 8 & & PHACE syndrome & $0 / 4$ \\
\hline Rhombencephalosynapsis & 6 & Dysgenesis of corpus callosum (2) & & $0 / 3$ \\
\hline & & Absence of septum pellucidum (1) & & \\
\hline & & Abnormal gyral pattern (1) & & \\
\hline Posterior fossa retrocerebellar cyst & 4 & Dysgenesis of corpus callosum (1) & & $0 / 1$ \\
\hline Joubert syndrome & 4 & Dysgenesis of the brainstem (2) & Joubert syndrome (2) & 1/2 (AHI1 mutation) \\
\hline Mega cisterna magna & 4 & & $\begin{array}{l}\text { Beckwith-Wiedemann } \\
\text { syndrome }\end{array}$ & $0 / 2$ \\
\hline
\end{tabular}

CNS, central nervous system; CHARGE, coloboma, heart defects, atresia of the choanae, retardation of growth and developmental delay, genital anomalies, ear anomalies; PHACE, posterior fossa, hemangioma, arterial lesions, cardiac abnormalities/aortic coarctation, eye abnormalities.

cisterna magna $(n=1)$. Conversely, global developmental delay (score $<2$ SDs below the normative mean) was documented in 19 children of our cohort. Specifically, gross and fine motor skills were most commonly affected (25/48 and 20/49 respectively), followed by cognitive (visual reception) and expressive language delays (19/49 for both). Out of the 48 children tested, 20 screened positive for early signs of autistic features, 12 demonstrated internalizing behavioral problems, while seven had externalizing behavior problems. Functional communication was impaired in 15 children, while 16 had function motor difficulties, 14 demonstrated impaired performance in daily living skills, and 10 experienced socialization problems. The outcomes of children with CBMs for each diagnostic category are summarized in Table II.

In a subgroup analysis of children with isolated CBMs compared with those with associated CNS findings, the latter group scored significantly lower on all our primary outcomes. Children with associated chromosome anomalies were not included in this subgroup analysis in order to isolate the effect of associated CNS anomalies on outcome. Developmental domains most significantly affected in children with concomitant CNS anomalies included global development, visual reception skills, and gross and fine motor skills. The detailed results are presented in Table III.

\section{Predictors of outcome}

The results of the multiple regression models showed that the presence of associated CNS findings and/or chromosome abnormalities were significant independent predictors of global development delay, gross and fine motor disabilities, and deficits in visual reception. Malformations that involved the cerebellar vermis were found to be a significant predictor of expressive language and gross motor deficits. The regression models were adjusted for the confounding effects of head circumference and age. Parameter estimates with their 95\% confidence intervals estimated from these regression models are presented in Table IV. The presence of CNS or chromosome anomalies was associated with an important reduction in the early learning composite score by 13.8 (95\% confidence interval 3.0-24.6). The effect of the presence of associated CNS findings and/or chromosome abnormalities on cognition, fine motor skills, and positive screening for ASD was also large (estimate of effect at least 8). Additionally, the presence of a malformation affecting the vermis had a most noticeable effect on expressive language and gross motor skills (estimates of effect of 6.8 and 7.9 respectively). A noteworthy finding was that the presence of shunts or the need for shunt revisions was not correlated with outcome. However, the presence of microcephaly was correlated with all functional outcome measures.

\section{DISCUSSION}

Several important findings are evident in this study. First, children with cerebellar anomalies have a high prevalence and broad spectrum of neurodevelopmental sequelae that have a negative impact on their QOL. Second, these deficits extend well beyond the motor domain to include cognitive, language, and social-behavioral impairments. Cerebellar malformations involving the vermis are associated with greater developmental disabilities compared with other cerebellar diagnostic groups. The presence of associated supratentorial anomalies and/or chromosomal abnormalities is significant independent determinants of neurodevelopmental disabilities. To our knowledge, this is one of the largest studies characterizing the spectrum of developmental and functional outcomes in young children with CBMs using standardized outcome measures.

Converging evidence from adult literature and older children with cerebellar injury supports an important role of the cerebellum in higher cognitive functions including cognitive, 
Table II: Developmental and functional outcomes for each clinical diagnostic category ${ }^{\mathrm{a}}$

\begin{tabular}{|c|c|c|c|c|c|c|c|c|c|}
\hline Outcome assessments ${ }^{\mathrm{a}}$ & $\begin{array}{l}\text { ICVH } \\
(n=17)\end{array}$ & $\begin{array}{l}\text { Bilateral } \\
\text { cerebellar } \\
\text { hypoplasia } \\
(n=8)\end{array}$ & $\begin{array}{l}\text { Vermis } \\
\text { hypoplasia } \\
(n=6)\end{array}$ & $\begin{array}{l}\text { DWM } \\
(n=5)\end{array}$ & $\begin{array}{l}\text { Unilateral } \\
\text { cerebellar } \\
\text { hypoplasia } \\
(n=4)\end{array}$ & $\begin{array}{l}\text { Rhombencep } \\
\text { halosynapsis } \\
(n=3)\end{array}$ & $\begin{array}{l}\text { Joubert } \\
\text { syndrome } \\
(n=2)\end{array}$ & $\begin{array}{l}\text { Mega } \\
\text { cisterna } \\
\text { magna } \\
(n=2)\end{array}$ & $\begin{array}{l}\text { PFC } \\
(n=2)\end{array}$ \\
\hline \multicolumn{10}{|l|}{ Mullen Scales of Early Learning } \\
\hline Early learning composite & 18 & 88 & 83 & 20 & 25 & 33 & 50 & 0 & 0 \\
\hline Fine motor & 24 & 63 & 83 & 40 & 25 & 67 & 50 & 0 & 0 \\
\hline Visual reception & 18 & 75 & 83 & 20 & 25 & 67 & 50 & 0 & 0 \\
\hline Expressive language & 12 & 75 & 100 & 25 & 0 & 67 & 100 & 0 & 0 \\
\hline Receptive language & 24 & 38 & 67 & 20 & 0 & 0 & 50 & 0 & 0 \\
\hline \multicolumn{10}{|l|}{ Peabody Developmental Motor Scales } \\
\hline Gross Motor & 24 & 100 & 100 & 0 & 50 & 100 & 100 & 50 & 0 \\
\hline Fine Motor & 18 & 63 & 100 & 0 & 25 & 33 & 50 & 50 & 0 \\
\hline \multicolumn{10}{|l|}{ Vineland Adaptive Behavior Scale } \\
\hline Communication & 18 & 63 & 67 & 0 & 25 & 33 & 50 & 0 & 0 \\
\hline Socialization & 12 & 25 & 67 & 0 & 0 & 33 & 50 & 0 & 0 \\
\hline Motor & 18 & 75 & 50 & 0 & 50 & 33 & 50 & 0 & 0 \\
\hline Daily living skills & 12 & 50 & 83 & 0 & 25 & 0 & 50 & 50 & 0 \\
\hline \multicolumn{10}{|l|}{ Child Behavior Checklist } \\
\hline Externalizing behaviors & 6 & 13 & 17 & 50 & 0 & 33 & 50 & 50 & 0 \\
\hline Internalizing behaviors & 24 & 50 & 17 & 25 & 25 & 0 & 50 & 50 & 0 \\
\hline Modified Checklist for Autism in Toddlers & 24 & 63 & 83 & 0 & 25 & 100 & 100 & 50 & 0 \\
\hline \multicolumn{10}{|l|}{ Pediatric Quality of Life Inventory } \\
\hline Total score & 18 & 50 & 50 & 0 & 25 & 67 & 50 & 50 & 0 \\
\hline
\end{tabular}

${ }^{a}$ All values are the percentages of children in each category scoring $<2 \mathrm{SDs}$ below the normative mean. ICVH, inferior cerebellar vermis hypoplasia; DWM, Dandy-Walker malformation; PFC, posterior fossa retrocerebellar cyst.

Table III: Relation between primary developmental and functional outcomes in children with associated CNS anomalies compared with those with isolated cerebellar malformations (CBMs)

\begin{tabular}{|c|c|c|c|c|}
\hline & \multicolumn{2}{|c|}{ Associated CNS anomalies ( $n=11)$} & \multicolumn{2}{|c|}{ Isolated CBMs ( $n=30)$} \\
\hline & Mean (SD) & $\begin{array}{l}\text { Percentage } \\
\text { below the 2SD } \\
\text { cut-off }\end{array}$ & Mean (SD) & $\begin{array}{l}\text { Percentage } \\
\text { below the 2SD } \\
\text { cut-off }\end{array}$ \\
\hline \multicolumn{5}{|c|}{ Mullen Scales of Early Learning } \\
\hline Early learning composite & $60.6(15.2)$ & 73 & $82.9(18.2)$ & 23 \\
\hline Expressive language & $26.3(8.1)$ & 64 & $37.2(11.2)$ & 26 \\
\hline Receptive language & $28.4(10.5)$ & 55 & $39.3(9.0)$ & 13 \\
\hline Visual reception & $26.4(9.8)$ & 73 & $41.7(13.0)$ & 23 \\
\hline \multicolumn{5}{|c|}{ Peabody Developmental Motor Scales } \\
\hline Gross motor & $65.8(1.8)$ & 91 & $79.4(12.2)$ & 33 \\
\hline Fine motor & $68.4(6.5)$ & 73 & $81.8(9.4)$ & 13 \\
\hline \multicolumn{5}{|c|}{ Modified Checklist for Autism in Toddlers } \\
\hline Total score & N/A & 82 & N/A & 23 \\
\hline
\end{tabular}

Results of independent sample $t$-test (Mullen Scales of Early Learning and Peabody Developmental Motor Scales) and $\chi^{2}$ (Modified Checklist for Autism in Toddlers) showed statistically significant differences in means for all outcomes $(p<0.01)$. CNS, central nervous system; N/A, not applicable.

language, and social skills. ${ }^{414}$ Despite this accruing evidence, a recent systematic literature review emphasized that outcome studies in children with CBMs have frequently been in the form of case series (39\%), conducted retrospectively, and focused predominantly on neurologic and cognitive impairments. Interestingly, only one-quarter of reports incorporated standardized outcome measures. ${ }^{5}$

Available outcome data in children with CBMs are, overall, conflicting. ${ }^{5}$ Our study corroborates previous reports of a significantly more favorable outcome in children with isolated
ICVH, posterior fossa retrocerebellar cyst, and mega cisterna magna, compared with children with Joubert syndrome or rhombencephalosynapsis. ${ }^{2,5,15}$ The outcome of children with DWM remains controversial, ${ }^{5,16,17}$ although our numbers were relatively small, the children with DWM in our study had a generally more favorable outcome than those in previous reports. ${ }^{15-17}$ Although some consider ICVH and mega cisterna magna as normal variants, our data corroborate recent studies that report that ICVH and mega cisterna magna are associated with mild functional deficits in a subset of 


\begin{tabular}{|c|c|c|c|c|c|c|c|c|}
\hline & \multicolumn{4}{|c|}{ Presence of CNS or chromosome anomalies } & \multicolumn{4}{|c|}{ Presence of cerebellar vermis malformation } \\
\hline & & \multicolumn{2}{|c|}{$\begin{array}{c}\text { Confidence } \\
\text { interval }\end{array}$} & \multirow[b]{2}{*}{$p$-value } & \multirow[b]{2}{*}{ Estimate of effect ${ }^{a}$} & \multicolumn{2}{|c|}{$\begin{array}{c}\text { Confidence } \\
\text { interval }\end{array}$} & \multirow[b]{2}{*}{$p$-value } \\
\hline & Estimate of effect ${ }^{a}$ & $\begin{array}{l}\text { Lower } \\
\text { limit }\end{array}$ & $\begin{array}{l}\text { Upper } \\
\text { limit }\end{array}$ & & & $\begin{array}{l}\text { Lower } \\
\text { limit }\end{array}$ & $\begin{array}{l}\text { Upper } \\
\text { limit }\end{array}$ & \\
\hline \multicolumn{9}{|c|}{ Mullen Scales of Early Learning } \\
\hline Early learning composite & -13.8 & -24.6 & -3.0 & 0.01 & -5.3 & -16.2 & 5.6 & 0.33 \\
\hline Expressive language & -6.4 & -12.9 & 0.0 & 0.05 & -6.8 & -13.3 & -0.0 & 0.04 \\
\hline Receptive language & -6.5 & -13.1 & 0.1 & 0.05 & -1.2 & -7.9 & 5.5 & 0.71 \\
\hline Visual reception & -8.5 & -16.1 & -0.9 & 0.03 & -5.6 & -13.3 & 2.1 & 0.15 \\
\hline \multicolumn{9}{|c|}{ Peabody DevelopmentalMotor Scales } \\
\hline Gross motor & -7.4 & -13.6 & -1.2 & 0.02 & -7.9 & -14.2 & -1.6 & 0.02 \\
\hline Fine motor & -8.0 & -13.5 & -2.5 & 0.01 & -1.9 & -7.5 & 3.7 & 0.50 \\
\hline \multicolumn{9}{|c|}{ Modified Checklist for Autism in Toddlers } \\
\hline Total score & $8.0^{\mathrm{b}}$ & 1.8 & 35.0 & 0.01 & 2.8 & 0.6 & 12.8 & 0.18 \\
\hline
\end{tabular}

${ }^{a}$ Estimate of effect: relation between predictor and functional outcome. ${ }^{b}$ Odds ratio and confidence interval for odds ratio of logistic regression. CNS, central nervous system.

children. ${ }^{2,18}$ Long-term studies are needed to confirm this observation. Similar to our study, in which the presence of supratentorial abnormalities was an important independent predictor of outcome, others have shown that children with isolated CBMs have a better outcome than their counterparts with associated CNS anomalies. ${ }^{5,16,17}$ In our study, the large differences in mean scores between the two groups highlight clinically important differences and support the role of supratentorial abnormalities in outcome determination. For example, differences in raw scores $>10$ points on the PDMS have been used to represent a clinically significant change. ${ }^{19}$ In this study, children with associated CNS anomalies compared with those with isolated CBMs obtained mean score differences $>13$ points on both the gross and fine motor scales. In addition, mean score differences on the Mullen represented a difference of nearly $1 \mathrm{SD}$ between the two groups for the early learning composite and over half an SD for all subtests.

The specific contribution of cerebellar vermis malformations on neurodevelopmental outcome has also been the focus of recent investigations. Developmental and acquired injuries of the vermis have been associated with cognitive, gross motor, and language impairments. $4,16,17,20,21$ The functional deficits associated with vermis lesions are also supported by a recent study demonstrating the presence of important intrinsic connections between the vermis and multiple cerebral regions involved in cognition, language, and emotions. ${ }^{22}$ Moreover, two studies have specifically examined the relation between the vermis and outcome in children with CBMs. ${ }^{16,17}$ Boddaert et al. ${ }^{16}$ compared the IQ of 21 children with DWM with and without normal vermis lobulation and showed that $82 \%$ in the former group had a normal IQ compared with none in the latter. Similarly, Klein et al. ${ }^{17}$ divided 26 children into two groups, one with partial agenesis of the vermis with normal lobulation, and a second with severe vermis malformations. In the former group, most (90\%) had a normal IQ and developmental quotients compared with none in the latter. However, an important confounder in both studies was concomitant supratentorial malformations in all children with an abnormal vermis as opposed to none in their comparison group; therefore the results cannot be precisely linked to the vermis. Although we did not evaluate vermis lobulation, our findings also highlight the fact that children with isolated vermis hypoplasia, DWM, and bilateral cerebellar hypoplasia experience a significantly higher prevalence of disabilities than those with isolated ICVH.

Our data also call attention to a high prevalence of cognitive dysfunction (i.e. visual reception and language) as well as social, behavioral, and affective problems. Interestingly, the prevalence and nature of developmental and functional disabilities in children with CBM parallels that of extreme preterm survivors of cerebellar hemorrhagic injury in early life, previously described by our group. ${ }^{4}$ In that study of ex-preterm children with isolated injury to the cerebellum, a high prevalence of cognitive (40\%) and language impairments (43\%), as well as social and behavior (26\%) problems, was reported. ${ }^{4}$ Moreover, a high rate of positive autism screening (43\%) was also described. ${ }^{4}$ In our cohort, CBMs were associated with a high rate (42\%) of early signs of autistic features, particularly among those children with a diagnosis of Joubert syndrome, rhombencephalosynapsis, bilateral cerebellar hypolasia, and vermis hypoplasia. It remains unclear, however, whether the reported high prevalence of a positive autism screening will translate into a diagnosis of ASDs or if these initial findings are transient or represent other forms of social-behavioral dysfunction not related to autism. In the general population, available data on the M-CHAT demonstrate that this screening tool has a positive predictive value of 0.68 (proportion of children with positive [autism screening] test results who will have a diagnosis of ASDs) and 0.79, and a negative predictive value of 0.99 and 0.99 (proportion of children who had negative [autism screening] test results who will not have a diagnosis of ASDs) for the 23 items and for the six critical items respectively. ${ }^{11}$ Standardized diagnostic tests for ASDs are 
needed in our present cohort to delineate the relative contribution of ASDs in this population. These studies are currently underway.

Adults with cerebellar injury and older children following cerebellar tumor resection have been reported to experience emotional dysregulation in the form of impaired behavioral modulation and flattening or disinhibition of affect. ${ }^{23}$ In addition, obsessive-compulsive traits may be prominent, as well as behavioral stereotypies and disturbed interpersonal relations that meet criteria for autism. ${ }^{10}$ Of particular note is that these affective and behavioral manifestations are most prominent when the vermis and paravermian regions of the cerebellum are decreased in volume. ${ }^{24}$ Furthermore, reports of patients with autism have shown well-defined cerebellar anatomic abnormalities, particularly hypoplasia of selective vermian lobules. ${ }^{25}$ Taken together, these data suggest a critical role of the cerebellar vermis for normal social-behavioral and affective skills.

To our knowledge, this study is the first of its kind to evaluate QOL in young children with CBMs. Although, measuring QOL has been recognized as an important factor evaluating the need for healthcare services or the effectiveness of healthcare interventions, ${ }^{26}$ no report so far has described QOL in this population. Our data show that approximately one-third of children with CBMs show impaired QOL and that lower developmental and functional scores significantly predict lower QOL.

Although this study has established an urgent need for better outcome definition in young survivors with CBMs, its limitations deserve mention. First, the cross-sectional design did not allow for observation of developmental progress over time. Moreover, although this study is one of the largest samples of children with CBMs ever reported, the small number of children in the various cerebellar diagnostic groups precluded further statistical analysis for each diagnostic category. Although the study described QOL in this population, it is important to note that this was performed by parent-proxy, which may have over- or underestimated the 'true' QOL in this population. Finally, despite the various contemporary frameworks for the classification of CBMs that have been proposed, there is still no universally accepted classification scheme for these malformations. As a result, we elected to use conventional diagnostic categories to describe our cohort. It remains unclear, however, whether using traditional diagnostic categories is the most appropriate way of categorizing CBMs to assist clinicians more effectively with prognostication.

In summary, we have demonstrated that CBMs are associated with a high prevalence of neurologic, developmental, and social-behavioral impairments, which translate into important functional disabilities in day-to-day life and poor QOL in many survivors. Greater understanding of the prevalence and extent of developmental disabilities associated with CBMs, based on the results of standardized outcome measures, will allow more accurate counseling of families and the establishment of targeted early intervention strategies. This will minimize long-term developmental morbidity and optimize QOL in these children. These advances can only be facilitated by large, multicenter, prospective studies using serial and quantitative MRI and standardized outcome measures that capture the scope of neurodevelopmental impairments and disabilities in young children with CBMs.

\section{ACKNOWLEDGEMENT}

This study was supported by the Lifebridge Fund, the Caroline Levine Foundation, and the Trust Family Foundation. M-EB received support from a McGill University Health Centre Research Institute Scholarship. CL (Canada Research Chair in Brain and Development [Tier II]) is supported by the Canada Research Chairs Program. AJDP is supported through a National Institutes of Health grant (1K24NS057568-01). We are indebted to the children and their families who participated in this study.

\section{REFERENCES}

1. Luciani L, editor. The cerebellum: new studies of normal and pathological physiology IX.Il. Florence, Italy: Xxxxx, 1891. Coi tipi dei successori Le Monnier [In Italian]

2. Limperopoulos C, Robertson RL, Estroff JA, et al. Diagnosis of inferior vermian hypoplasia by fetal magnetic resonance imaging: potential pitfalls and neurodevelopmental outcome. Am 7 Obstet Gynecol 2006; 194: 1070-6.

3. Schmahmann JD, Pandya DN. The cerebrocerebellar system. Int Rev Neurobiol 1997; 41: 31-60.

4. Limperopoulos C, Bassan H, Gauvreau K, et al. Does cerebellar injury in premature infants contribute to the high prevalence of long-term cognitive, learning, and behavioral disability in survivors? Pediatrics 2007; 120: 584-93.

5. Bolduc M-E, Limperopoulos C. Neurodevelopmental outcomes in children with cerebellar malformations: a systematic review. Dev Med Child Neurol 2009; 51: 256-67.

6. Barkovich AJ. Pediatric neuroimaging. 4th edn. Philadelphia: Lippincott Williams \& Wilkins, 2005.

7. Mullen EM. Mullen Scales of Early Learning. AGS edn. Circle Pines, MN: American Guidance Service, 1995.
8. Folio RM, Fewell RR. Peabody Developmental Motor Scales and Activity Cards. Austin, TX: DLM Teaching Resources, 1983.

9. Sparrow SS, Balla DA, Cicchelli DV. Vineland Adaptive Behavior Scales. Interview edn, extended from manual. Circle Pines, MN: American Guidance Service, 1984.

10. Achenbach TM, Rescorla L. Manual for the Child Behavior Checklist. Burlington, VT: Department of Psychiatry, University of Vermont, 2000.

11. Robins D, Fein D, Barton M. The Modified Checklist for Autism in Toddlers (M-CHAT). Storrs, CT: University of Connecticut, 1999.

12. Varni JW, Seid M, Kurtin PS. PedsQL 4.0: reliability and validity of the Pediatric Quality of Life Inventory Version 4.0 Generic Core Scales in healthy and patient populations. Med Care 2001; 39: 800-12.

13. Hollingshead AB. Four Factor Index of Social Status. New Haven, CT: Yale Station, 1965.

14. Schmahmann JD. The cerebellum and cognition. San Diego, CA: Academic Press, 1997.
15. Forzano F, Mansour S, Ierrullo A, Homfray T, Thilaganathan B. Posterior fossa malformation in fetuses: a report of 56 further cases and a review of the literature. Prenat Diagn 2007; 27: 495-501.

16. Boddaert N, Klein O, Ferguson N, et al. Intellectual prognosis of the Dandy-Walker malformation in children: the importance of vermian lobulation. Neuroradiology 2003; 45: $320-4$.

17. Klein O, Pierre-Kahn A, Boddaert N, Parisot D, Brunelle F. Dandy-Walker malformation: prenatal diagnosis and prognosis. Childs Nerv Syst 2003; 19: 484-9.

18. Zimmer EZ, Lowenstein L, Bronshtein M, Goldsher D, Aharon-Peretz J. Clinical significance of isolated mega cisterna magna. Arch Gynecol Obstet 2007; 276: 487-90.

19. Palisano RJ, Kolobe TH, Haley SM, Lowes LP, Jones SL. Validity of the Peabody Developmental Gross Motor Scale as an evaluative measure of infants receiving physical therapy. Phys Ther 1995; 75: 939-48.

20. Riva D, Giorgi C. The cerebellum contributes to higher functions during development: evidence from a series of chil- 
dren surgically treated for posterior fossa tumors. Brain 2000; 123: 1051-61.

21. Schmahmann JD. Disorders of the cerebellum: ataxia, dysmetria of thought, and the cerebellar cognitive affective syndrome. 7 Neuropsycbiatry Clin Neurosci 2004; 16: 367-78.

22. Habas C, Kamdar N, Nguyen D, et al. Distinct cerebellar contributions to intrinsic connectivity networks. 7 Neurosci 2009; 29: 8586-94.
23. Schmahmann JD, Weilburg JB, Sherman JC. The neuropsychiatry of the cerebellum - insights from the clinic. Cerebellum 2007; 6: 254-67.

24. Hallahan B, Daly EM, McAlonan G, et al. Brain morphometry volume in autistic spectrum disorder: a magnetic resonance imaging study of adults. Psychol Med 2008; 8: 1-10.
25. Courchesne E, Yeung-Courchesne R, Press GA, Hesselink JR, Jernigan TL. Hypoplasia of cerebellar vermal lobules VI and VII in autism. N Engl 7 Med 1988; 318: 1349-54.

26. Carr AJ, Higginson IJ. Are quality of life measures patient centred? BMF 2001; 322: 1357-60.

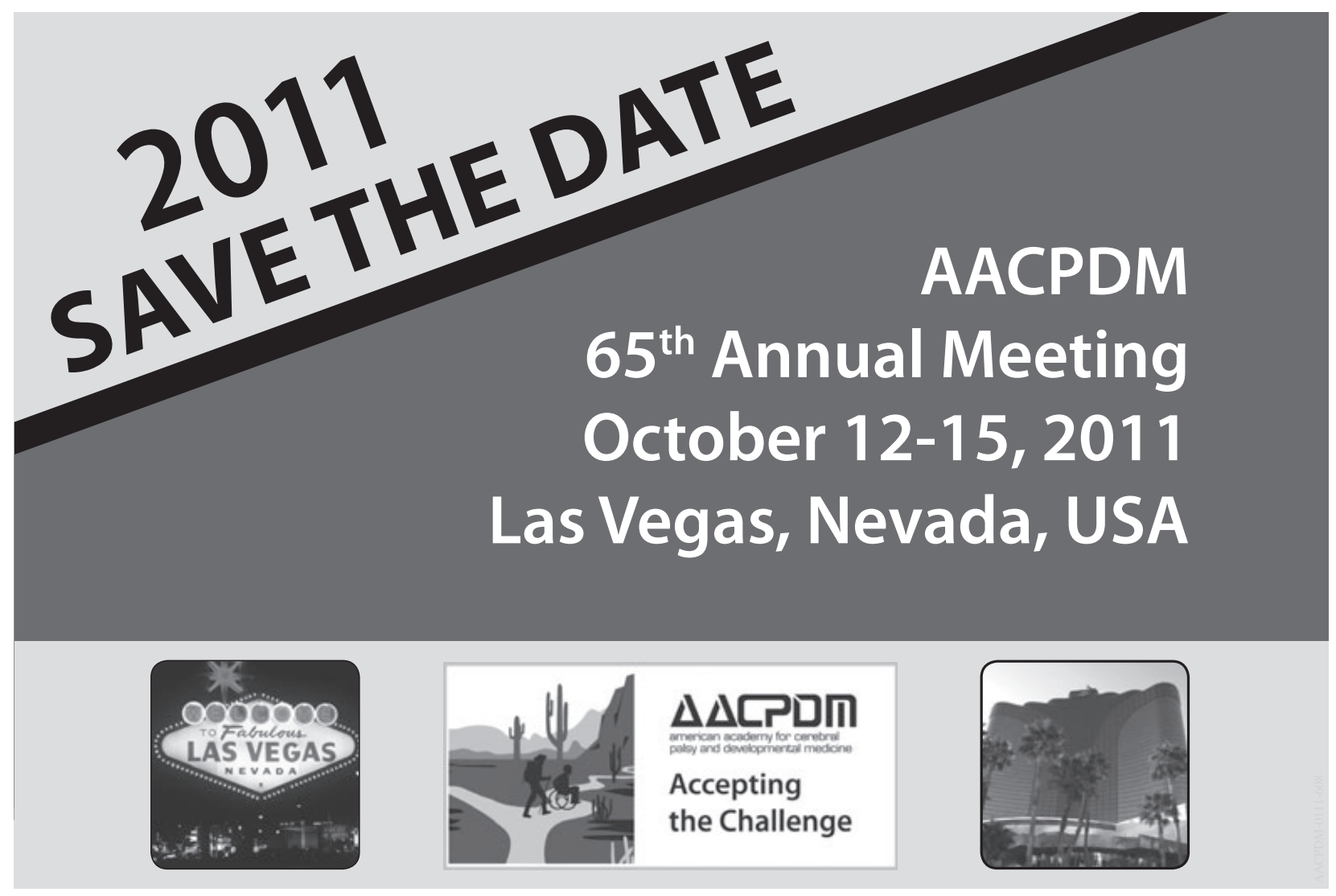

